\title{
Identification and characterization of cancer stem cells in human head and neck squamous cell carcinoma
}

\author{
Jing Han ${ }^{1}$, Toshio Fujisawa ${ }^{2}$, Syed R Husain ${ }^{1}$ and Raj K Puri ${ }^{\text {* }}$
}

\begin{abstract}
Background: Current evidence suggests that initiation, growth, and invasion of cancer are driven by a small population of cancer stem cells (CSC). Previous studies have identified CD44+ cells as cancer stem cells in head and neck squamous cell carcinoma (HNSCC). However, CD44 is widely expressed in most cells in HNSCC tumor samples and several cell lines tested. We previously identified a small population of CD24+/CD44+ cells in HNSCC. In this study, we examined whether this population of cells may represent CSC in HNSCC.

Methods: CD24+/CD44+ cells from HNSCC cell lines were sorted by flow cytometry, and their phenotype was confirmed by qRT-PCR. Their self-renewal and differentiation properties, clonogenicity in collagen gels, and response to anticancer drugs were tested in vitro. The tumorigenicity potential of CD24+/CD44+ cells was tested in athymic nude mice in vivo.

Results: Our results show that CD24+/CD44+ cells possessed stemness characteristics of self-renewal and differentiation. CD24+/CD44+ cells showed higher cell invasion in vitro and made higher number of colonies in collagen gels compared to CD24-/CD44+ HNSCC cells. In addition, the CD24+/CD44+ cells were more chemo-resistant to gemcitabine and cisplatin compared to CD24-/CD44+ cells. In vivo, CD24+/CD44+ cells showed a tendency to generate larger tumors in nude mice compared to CD24-/CD44+ cell population.
\end{abstract}

Conclusion: Our study clearly demonstrates that a distinct small population of CD24+/CD44+ cells is present in HNSCC that shows stem cell-like properties. This distinct small population of cells should be further characterized and may provide an opportunity to target HNSCC CSC for therapy.

Keywords: HNSCC (head \& neck squamous cell carcinoma), Stem-like cells, CD24, CD44, Salivary gland malignant neoplasms

\section{Background}

Squamous cell carcinoma of head and neck (HNSCC) is a heterogeneous disease [1]. Although recent advances in treatment have improved quality of life, overall 5 year survival rates have not improved significantly [2]. HNSCC frequently shows local recurrence and metastasis after the initial treatment of the primary tumor [3]. Mortality from this disease remains high because of the development of metastases and therapy-resistant local and regional

\footnotetext{
* Correspondence: raj.puri@fda.hhs.gov

${ }^{1}$ Tumor Vaccines and Biotechnology Branch, Division of Cellular and Gene Therapies, Center for Biologics Evaluation and Research, Food and Drug Administration, NIH Bldg 29B, Rm 2NN20, 29 Lincoln Dr., Bethesda, MD 20892, USA

Full list of author information is available at the end of the article
}

recurrences [1]. Progress in treatment and prognosis for HNSCC has been limited and the molecular mechanisms of HNSCC escape from chemo- and/or radiation therapies remain mostly unknown.

Recent evidence suggests that small populations of tumor-initiating cells or cancer stem cells (CSC) are responsible for initiation, tumorigenesis, progression, and metastasis [4]. CSCs undergo self-renewal and differentiation to yield phenotypically diverse non-tumorigenic and tumorigenic cancer cells $[4,5]$. CSCs have been identified, isolated, and characterized in various types of cancers, such as leukemia [6], brain tumor [7], colorectal cancer [8], ovarian cancer [9], bladder cancer [10], pancreatic cancer [11] and others. It has been postulated that CSCs 
within the bulk tumor may escape conventional therapies, thus leading to disease relapse. Therefore, an important goal of therapy could be to identify and kill this CSC population. If CSCs can be identified prospectively and isolated, then we should be able to identify new diagnostic markers and potential therapeutic targets.

HNSCCs are heterogeneous in cellular composition. A CD44+ subpopulation of cells with CSC properties was first identified in HNSCC [12]. These CD44+ cells express a high level of the BMI1 gene, which has been demonstrated to play a role in self-renewal and tumorigenesis $[13,14]$. In addition to CD44, other putative stem cell markers reported to be present in HNSCC cell lines include CD29 and CD133, but the proportion of cells expressing these markers differed from one cell line to the other [15]. Additional studies indicate that ALDH activity may represent a more specific marker for CSCs in HNSCC [16,17]. It is unknown if cancer stem cell markers are tumor specific for the tissue of origin or for the niche where the tumor is growing [18].

The CD24 gene has raised considerable interest in tumor biology. A large body of literature suggests a role for CD24 in tumorigenesis and tumor progression. CD24 expression causes the acquisition of multiple cellular properties associated with tumor growth and metastasis [19]. Recent studies have identified CD24 as a marker in cancer stem cells in several cancers, including pancreatic cancer [11], colorectal cancer-derived cell lines [8], and ovarian cancer [9]. Cancer stem cell immunophenotype studies in oral squamous cell carcinoma indicated that patients with CD24 and CD44 double-positive cells showed the lowest overall survival rate compared to other immunophenotypes [20]. In our previous studies, we also found that a small population of CD24+/CD44+ cells existed in HNSCC [21]. Whether or not CD24+/CD44+ cells represent a potential phenotype of cancer stem cells in HNSCC remains to be determined.

In the present study, we have isolated the CD24+/CD44+ population from HNSCC cell lines and determined whether this cell population has cancer stem cell properties by a variety of different approaches. We demonstrate that the $\mathrm{CD} 24+/ \mathrm{CD} 44+$ population indeed has CSC properties in HNSCC and this population should be further characterized.

\section{Methods}

\section{Cell cultures}

HNSCC cell line A253 (ATCC HTB-41) was obtained from American Type Culture Collection (ATCC, Manassas, VA). HNSCC cell line KCCT873 was obtained from Yokohama City University Hospital [22]. A253 cells were established from tumor originated from submaxillary salivary gland. KCCT873 cells were originated from tongue tumor. A253 cells were grown in McCoy's Modified Medium, and
KCCT873 cells in RPMI 1640 medium. Cell culture media were supplemented with $10 \%$ fetal bovine serum and $1 \%$ penicillin/streptomycin (Lonza, Walkersville, MD). The cells were maintained at $37^{\circ} \mathrm{C}$ in a humidified atmosphere containing $5 \% \mathrm{CO}_{2}$.

\section{Fluorescent-activated cell sorting and flow cytometry analysis}

Cell sorting by flow cytometry was performed by $\mathrm{Mr}$. Howard Mostowski at the Flow Cytometry Core facility, Center for Biologics Evaluation and Research, FDA. Cells were labeled with mouse anti-human CD44-PE (Millipore, Temecula, CA) and mouse anti-human CD24-FITC (Santa Cruz Biotech, Santa Cruz, CA) antibodies. The top or bottom cells in the 0.5 to 1 percentile fluorescence intensity of each CD24+/CD44+ and CD24-/CD44+ subpopulations were sorted and collected separately for further experiments.

For flow cytometric analysis of other markers, cells $\left(10^{6}\right.$ cells $\left./ \mathrm{ml}\right)$ were resuspended and incubated with various antibodies, CD29-APC, CD73-APC, and CD90PerCP-Cy5.5 (eBioscience - www.ebioscience.com), CD24FITC (Santa Cruz Biotech), and CD44-PE (Millipore), according to the manufacturer's instructions for $30 \mathrm{~min}$ on ice, washed with PBS three times, and fixed with $1 \%$ paraformaldehyde for later analysis. For controls, relevant isotype control antibody (eBioscience) and no antibody was used in parallel. Data were analyzed using FlowJo software (Tree Star Inc., Ashland, OR).

\section{Real-time PCR}

For qRT-PCR, total RNAs was extracted by Trizol reagent according to the manufacturer's instructions (Invitrogen, Carlsbad, CA). The $1^{\text {st }}$ strand cDNA was synthesized from $1 \mu \mathrm{g}$ of total RNA using Superscript II Reverse Transcriptase (Invitrogen) according to manufactures specifications. The resulting cDNA was amplified by using gene-specific primers. The primer sequences for each tested gene are listed in Additional file 1: Table S1. For amplification, samples were prepared with SsoAdvanced ${ }^{\mathrm{TM}}$ SYBR $^{\circ}$ Green Supermix (Bio-Rad) following the manufacture's protocol, and run on a Bio-Rad CFX96 Touch ${ }^{\mathrm{TM}}$ Real-Time Detection System. Buffer only and no template were included in each assay run as controls. All samples and controls were run in triplicate. Gene-specific amplification was normalized to $\beta$-Actin and relative fold change was calculated following the manufacture's protocol (Bio-Rad).

\section{Cell proliferation assay}

One thousand sorted cells per well were cultured in quadruplicate in 96-well plates for the indicated period of time. Cell proliferation was detected by using CellTiter-Glo ${ }^{\circ}$ Luminescent Cell Viability Assay kit (Promega, Madison, WI). Cell viability was quantified by measuring 
the absorbance using a microplate reader (Molecular Devices, Sunnyvale, CA) with $500 \mathrm{~ms}$ integration. Experimental background was determined by using empty wells with medium.

\section{Colony-forming assay}

Collagen type I gels were prepared with cell culture medium to make final collagen concentration of $2 \mathrm{mg} / \mathrm{mL}$ $(\mathrm{pH}=7.0)$ [23]. For cell cultures within collagen gels, $1.5 \mathrm{~mL}$ cell suspension (500 cells $/ \mathrm{mL}$ ) was mixed with $1.5 \mathrm{~mL}$ of collagen solution. The mixture was plated in six-well plates, and placed in $37^{\circ} \mathrm{C}$ incubator for gelation. After gelation, the collagen gels were overlaid with $3 \mathrm{~mL}$ of complete medium and incubated in a humidified atmosphere containing 95\% air and 5\% $\mathrm{CO}_{2}$. Cells were cultured for six days. Cell colonies were visualized with Coomassie Blue solution staining (0.5\% Coomassie Brilliant Blue G250, Bio-Rad), and visible colonies were counted. Assays were performed in triplicate.

\section{Matrigel invasion assay}

Cell invasion was studied by using BD BioCoat Matrigel invasion chambers (BD Biosciences; 24-well, $8 \mu \mathrm{m}$ pore size) with $10 \%$ fetal bovine serum as a chemo attractant, and following the manufacture's protocol. Briefly, one thousand cells were loaded into the chamber and incubated for 24 to $72 \mathrm{hrs}$ at $37^{\circ} \mathrm{C}$. Noninvasive cells were removed from the upper surface of the membrane with a cotton swab, and cells on the bottom surface of the membrane were fixed and stained with H\&E. Cells in five random fields per well were counted. The experiments were performed in duplicate.

\section{Drug sensitivity assay}

Following cell sorting, both CD24+/CD44+ and CD24-/ CD44+ cells were cultured for 2 days to eliminate damaged cells caused by the sorting process. Cells were then plated at a density of $1 \times 10^{3} /$ well in 96-well plates. Chemotherapeutic reagents, Gemcitabine or Cisplatin, were added to the cells at gradually increasing concentrations. The cells were cultured for $72 \mathrm{hrs}$, and the cell viability was determined by CellTiter-Glo Assay (Promega, Madison, WI) according to the manufacturer's protocol.

\section{Tumor xenograft studies}

Animal studies were conducted under a CBER ACUCapproved protocol in accordance with the principles and procedures outlined in the NIH Guide for the Care and Use of Laboratory Animals. Female athymic nude immunodeficient mice between 4-to 6-week-age were obtained from the NCI Animal Facility (NCI-Frederick). Before injection, cells were re-suspended in a 1:1 mixture of Matrigel (BD Biosciences) and PBS. A 100- $\mu$ l cell suspension containing $100,1,000$, or 10,000 sorted CD24+ and CD24-cells was subcutaneously injected into the dorsal flank of each mouse. For the control groups, mice received $100 \mu \mathrm{l}$ injections of the parent unsorted cells in corresponding concentrations. Tumor size (major axis $\times$ the minor axis) was measured weekly after tumor

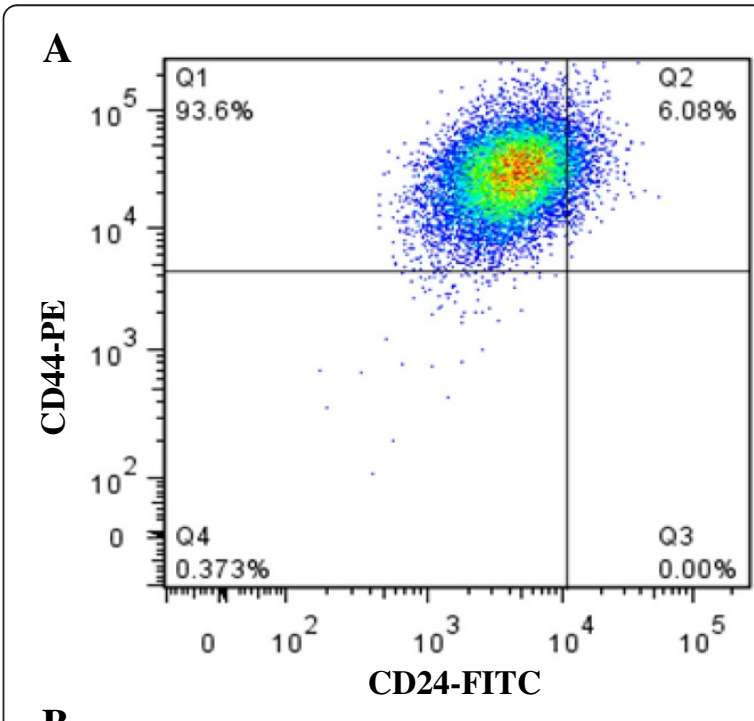

B

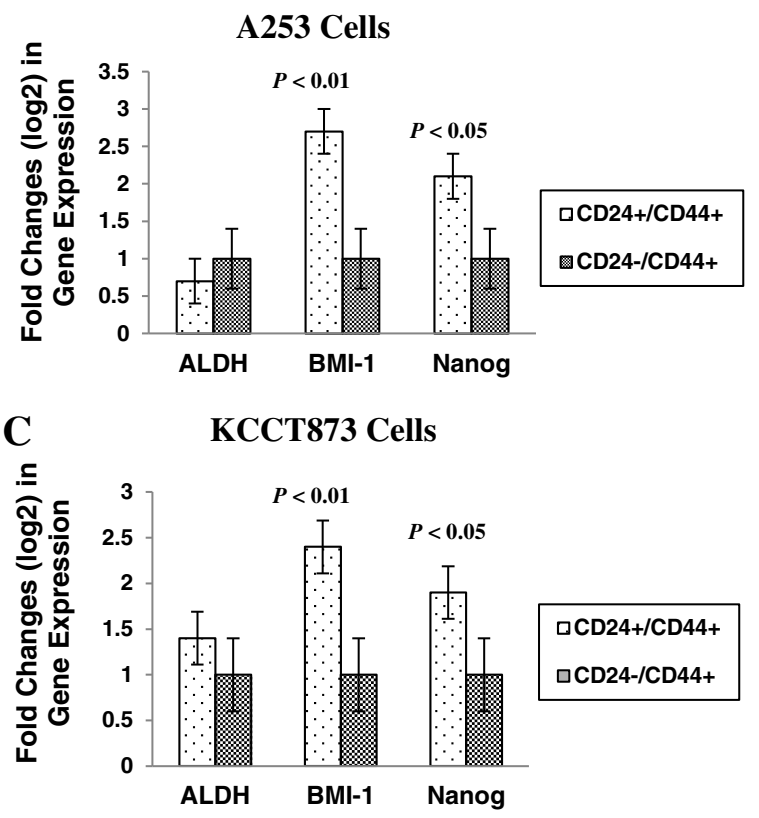

Figure 1 Expression of CD24 and CD44 in A253 HNSCC cells. (A) Flow cytometric analysis of CD24+ and CD44+ cells in A253 HNSCC cell line. Dual staining of A253 HNSCC cells indicate that CD24+/CD44+ subpopulation is $\sim 6 \%$, while CD24-/CD44+ subpopulation is $>93 \%$ in the whole cell population. qRT-PCR analysis of stemness-related genes in FACS-sorted CD24+/CD44+ and CD24-/CD44+ cells derived from A253 (B) and KCCT873 (C) tumor cells. Data represent log2 mean fold changes in gene expression \pm SD of triplicate determinations in CD24+/CD44+ compared to CD24-/CD44+ subpopulations from both cell lines. $P$ values for two genes, BMI1 and Nanog, in two cell lines are shown. 
challenge. Animal experiments were repeated several times. At the end of the experimental period, tumor tissues were collected and fixed in formalin for further immunohistochemical studies.

\section{Immunohistochemical studies of HNSCC tumor tissues} Immunohistochemical (IHC) studies of tumor sections were performed on formalin-fixed, paraffin-embedded tumors isolated from tumor xenografts in the study. Tissue sections were deparaffinized by xylene, and re-hydrated with sequential washes of $100 \%, 75 \%$, and $50 \%$ ethanol, and PBS. For antigen retrieval, slides were placed in $50 \mathrm{mM}$ citrate buffer $\mathrm{pH} 6.0$ (Vector Lab, CA), boiled for $5 \mathrm{~min}$, and stayed in the buffer for $15 \mathrm{~min}$. Endogenous peroxidase activity was inhibited with $3 \%$ hydrogen peroxidase in PBS. Non-specific binding was blocked with $2.5 \%$ normal serum and $1 \%$ bovine serum albumin (BSA) for
$1 \mathrm{hr}$. Tissue sections were incubated with various antibodies, CD24 and CD44 (Millipore), or isotype control (IgG) (Sigma) overnight at $4^{\circ} \mathrm{C}$. Immunodetection was performed using $\mathrm{ABC}$ staining systems according to manufacturer's instructions (Santa Cruz Biotech). All sections were counterstained with haematoxylin. After dehydration with washes of $95 \%$ and $100 \%$ ethanol and xylene, tissue sections with permanent mounting medium were covered with glass coverslips, and viewed by light microscope. H\&E staining was also performed on the section from each tumor tissue sample.

\section{Statistical analysis}

Statistical analyses were performed by paired Student's t-test between two groups. Data were presented as mean \pm SD. $P$ value of $<0.05$ was considered statistically
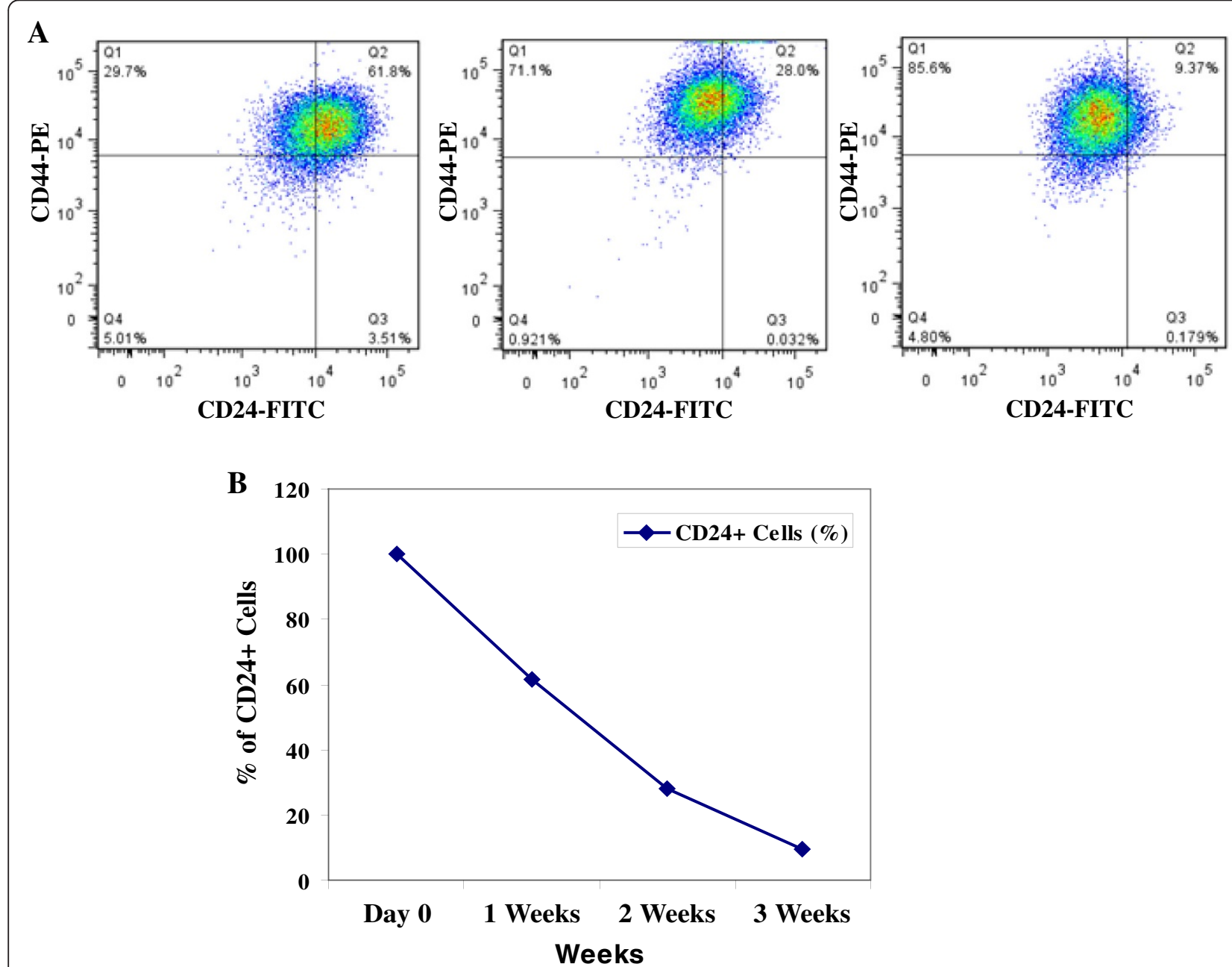

Figure 2 Differentiation of CD24+/CD44+ cells. (A) A253 CD24+ HNSCC cells differentiate into CD24-cells. Population dynamics modeled by a simple growth model in which CD24+ cells divide and switch to a CD24-state. Flow cytometry plots illustrate the sorted CD24+ cell populations at week one, two and three, from left to right panels. (B) Flow sorted CD24+ cells were monitored for 3 weeks in cell culture for their ability to convert into CD24-cells. Day 0 indicates the day cells were sorted by CD24 expression. The percentage of the CD24+ cells decreased in a time-dependent manner. 
significant. Each experiment was repeated at least twice including animal experiments.

\section{Results}

Isolation and characterization of CD24+/CD44+ cells in HNSCC cell lines

To determine the percentage of the putative cancer stemlike cells in the HNSCC cell population, cell suspensions from cell lines A253 and KCCT873 were analyzed and sorted for cell surface markers CD24 and CD44 by flow cytometry. Two phenotypic subpopulations were separated. CD24+/CD44+ cells were only $\sim 5-8 \%$ in whole cell population. In contrast, CD24-/CD44+ cells were $>90 \%$ in whole cell population of both HNSCC cell lines (Figure 1A).

We next investigated the expression of known "stemness" genes in the isolated CD24+/CD44+ and CD24-/ CD44+ subpopulations by real-time RT-PCR technology. We tested expression of six genes including ALDH1, BMI1, CD133, Nanog, Oct3/4, and Sox2. BMI1 and Nanog genes showed a significantly higher expression in CD24+/CD44+ compared to CD24-/CD44+ subpopulations from both HNSCC cell lines. However, there was no significant difference in ALDH1 expression between CD24+/CD44+ and CD24-/CD44+ subpopulations from both cell lines (Figure $1 \mathrm{~B}$ and $\mathrm{C}$ ). CD133 was only expressed in one cell line (KCCT873) at a very low level and did not show a clear difference between two subpopulations of cells (data not shown). A253 cells did not show any expression of CD133 gene. The expression of Oct $3 / 4$ and Sox 2 was absent in both cell subpopulations in both cell lines (data not shown).

\section{Cellular properties of CD24+/CD44+ cells in vitro}

To explore the self-renewal and differentiation capacity of CD24+/CD44+ cells, the purified CD24+/CD44+ cells were cultured in vitro for 3 weeks, and variations in CD24 expression were examined by flow cytometry. We found that the proportion of CD24+/CD44+ cells dramatically declined in a time dependent manner in the CD24+/CD44+ sorted population of cells. CD24+ cells in CD24+/CD44+ population decreased to $\sim 62 \%$ one week after culture and continued to decrease to $28 \%$ two weeks after cell culture. The proportion of the CD24+/CD44+ cells returned to similar presorting level $(<10 \%)$ after three weeks culture. In contrast, the proportion of CD24-/CD44+ cells in the cell population gradually increased from $\sim 30 \%$ at the first week to $~ 86 \%$ after three weeks, indicating that the CD24+/CD44+ cells give rise to CD24-/CD44+ cells (Figure 2A and B).

Cell proliferation assays indicated that the growth rate of CD24+/CD44+ cells was slightly lower compared to CD24-/CD44+ cells for up to 5 days after cell sorting (Figure $3 \mathrm{~A}$ and $\mathrm{B}$ ). These results indicate that
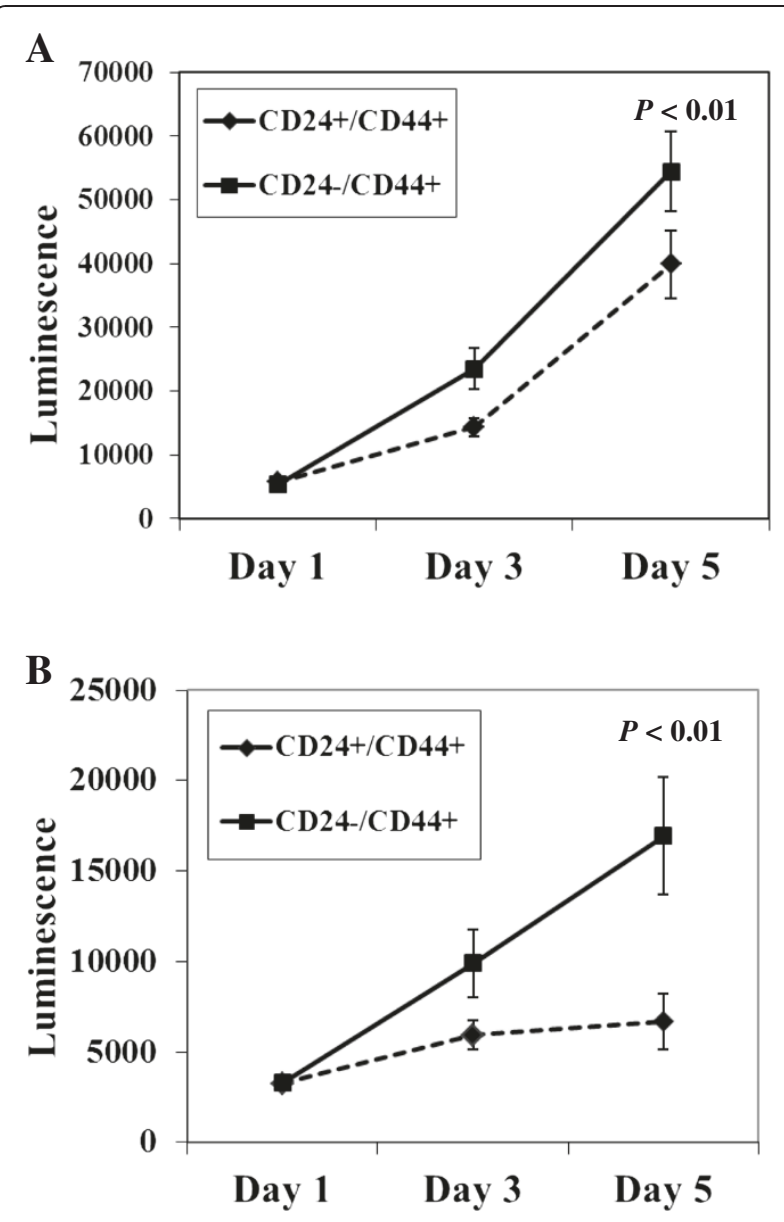

Figure $\mathbf{3}$ Cell proliferation assay. Cells were cultured in quadruplicate in a 96-well plate at a density of 1000 cells/per well, and proliferation was measured by Cell Titter-Glo cell viability assay. Growth curve of CD24+/CD44+ and CD24-/CD44+ subpopulations of A253 cells (A) and KCCT873 cells (B) are shown. Data represent mean \pm SD of triplicate determinations. $P$ value is shown for day 5 time point.

CD24+/CD44+ cells show asymmetric division-like proliferation pattern, indicating the self-renewal and differentiation potential to produce heterologous descendent CD24-/CD44+ cells in culture.

We next investigated the invasion ability of CD24+/ CD44+ and CD24-/CD44+ subpopulations by matrigel invasion assays. We observed that the number of invading cells in the CD24+/CD44+ cells was significantly higher compared to CD24-/CD44+ cells, indicating that CD24+/ CD44+ cells have higher invasion ability compared to CD24-/CD44+ cells $(\mathrm{p}<0.02$ for A253 and $\mathrm{p}<0.01$ for KCCT873 compared to CD24-/CD44+ cells) (Figure 4A).

The colony-formation capacity of CD24+/CD44+ and CD24-/CD44+ subpopulations was also tested. Our results indicate that CD24+/CD44+ cells form significantly higher number of colonies compared to CD24-/CD44+ cell subpopulation $(p<0.05)$ (Figure 4B). 

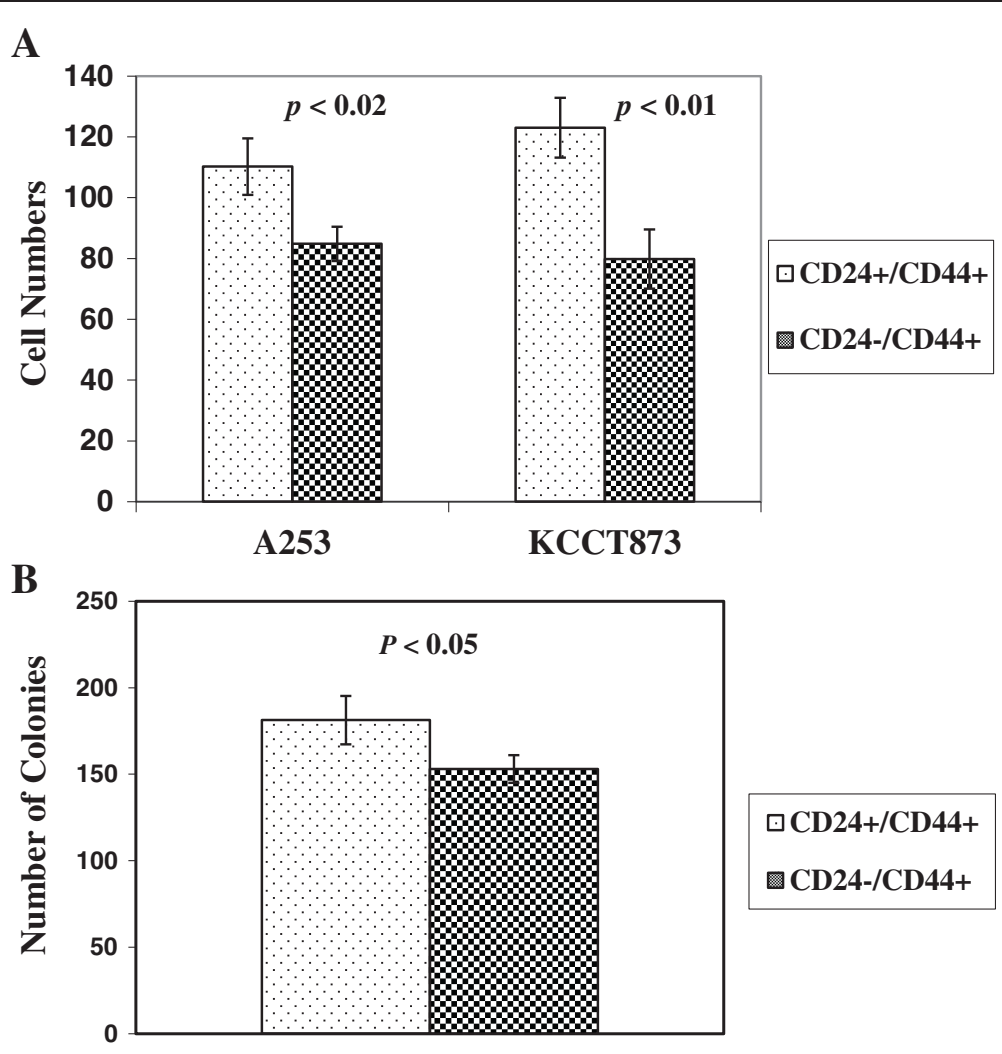

$\mathbf{A 2 5 3}$

Figure 4 Cell invasion and clonogenic assays. (A) Matrigel invasion activity of CD24+/CD44+ and CD24-/CD44+ flow cytometry-sorted cells from HNSCC cell lines. The number of cells invading through the Matrigel was assessed at $24 \mathrm{hr}$. (B) Colony-forming assay with FACS-sorted CD24+/CD44+ and CD24-/CD44+ cells. The CD24+/CD44+ cells show significantly higher number of colonies. $P$ values for invasion and clonogenic assays are shown in the figure.

\section{CD24+ cells show higher drug resistance to chemotherapeutic agents in vitro}

Cisplatin (cis-diammine-dichloroplatinum (II)) is used for treatment of a wide range of cancers, including head \& neck tumors. Cisplatin often leads to an initial therapeutic success associated with partial response or disease stabilization [24]. Gemcitabine is a nucleoside analog displaying a wide spectrum of antitumor activity [25]. Although both drugs have been used for chemotherapeutic treatment of patients with head \& neck tumors, many patients are intrinsically resistant to these drugs [24]. Recent studies have indicated that cancer stem cell phenotypes are associated with drug resistance to chemotherapeutic drugs [26,27]. To evaluate the drug resistance properties of FACS sorted HNSCC cells, CD24+/CD44+ and CD24-/CD44+ cells were grown and treated with various concentrations of either cisplatin or gemcitabine for 72 hours, and then cell survival was assessed by determining cell viability. CD24+/CD44+ cells seem to show small but significantly higher drug resistance to either chemotherapeutic agent when compared to CD24-/CD44+ cells (Figure 5). For example,
CD24+/CD44+ cells showed higher survival rate (53.5\%) compared to CD24-/CD44+ cells $(40 \%)$ when treated with $1000 \mathrm{nM}$ cisplatin $(p<0.01)$ (Figure 5A). Similarly, CD24+/CD44+ cells showed $>10 \%$ higher survival rate (37\%) compared to survival rate (26\%) of CD24-/ CD44+ cells when treated with $10 \mathrm{nM}$ gemcitabine $(p<0.01)$ (Figure 5B).

\section{Tumorigenicity of CD24+/CD44+ and CD24-/CD44+ subpopulations}

We next evaluated whether the two subpopulations (CD24+/CD44+ and CD24-/CD44+) of HNSCC cells were endowed with differential tumorigenic potential. Several independent experiments were performed with two different HNSCC cell lines. The two phenotypic subpopulations of cells, CD24+/CD44+ and CD24-/CD44+, were sorted by flow cytometry, suspended in a Matrigel mixture (1:1), and then S.C. injected into athymic nude mice. The tumor size was measured weekly for 9 weeks, at which time animals were sacrificed. When minimal $\left(1 \times 10^{2}\right)$ to maximal $\left(1 \times 10^{4}\right)$ numbers of cells per mouse were injected, both CD24+/CD44+ and CD24-/CD44+ 

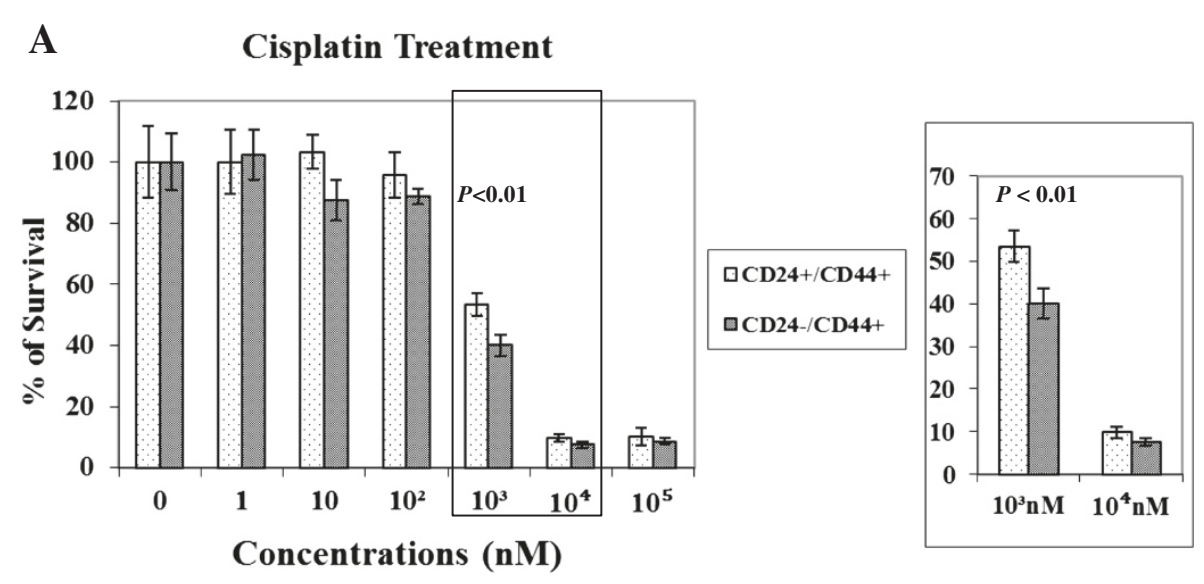

B

Gemcitabine Treatment
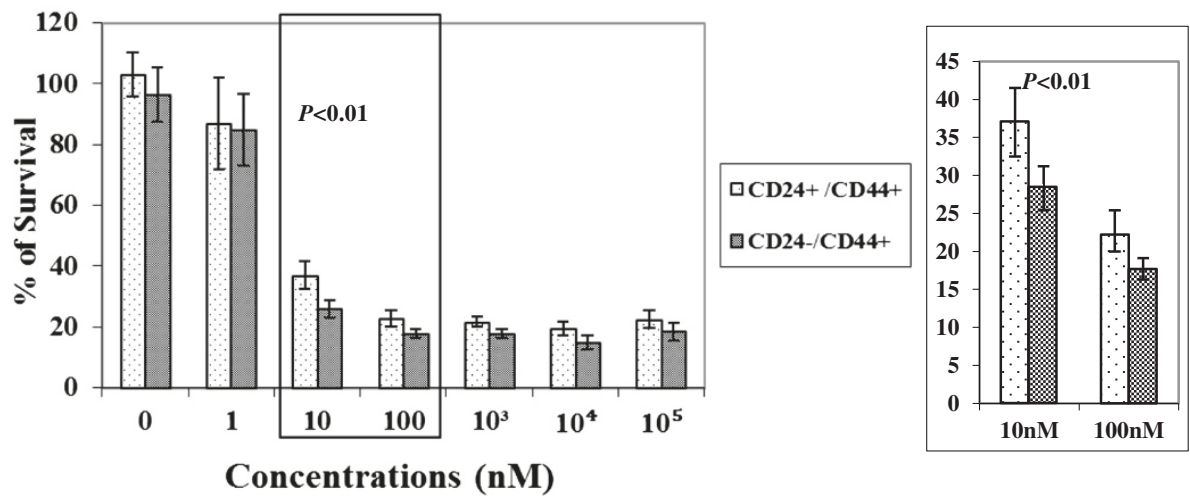

Figure 5 Sensitivity of CD24+/CD44+ and CD24-/CD44+ cells to cisplatin and gemcitabine anticancer drugs. Flow cytometry sorted cells were exposed to cisplatin (A), and gemcitabine (B) at increasing concentrations for $72 \mathrm{hr}$, followed by cell viability measurement by Cell Titter-Glo Cell Viability Assay. Differences in drug resistance between CD24+/CD44+ and CD24-/CD44+ cells were calculated. All experiments were performed in triplicate and data are shown as mean \pm SD. Data in inset show statistical significance at $p<0.01$ for both treatments.

cells formed tumors and thus were tumorigenic. However, the size of tumor generated by CD24+/CD44+ cells was significantly larger than the size of the tumors from CD24-/CD44+ or unsorted control cells (Figure 6) indicating CD24+/CD44+ cells are highly aggressive.

Immunohistochemical staining for CD24 and CD44 on tumor tissues isolated from tumor xenografts at the end of the study were performed to determine whether CD24+/CD44+ CSC maintained their phenotype at the end of the experiment. Upon H\&E staining, A253 cells showed submaxillary salivary gland features since these cells originated from submaxillary salivary gland tumor. KCCT873 cells showed similar features. By IHC, strong positive staining for $\mathrm{CD} 24$ was observed on the surface of salivary gland appearing structure in xenograft tumors derived from both cell lines. In addition, strong positive staining for CD44 was observed not only on the surface of salivary gland appearing structures, but also on the dense carcinoma cells within the tumor mass as well (Figure 7). Since xenograft tumors generated from both CD24+/CD44+ and CD24-/CD44+ cells showed the similar immunohistochemical staining, we hypothesized that the CD24+/CD44+ cells may have been generated during the in vivo tumor growth from CD24-/CD44+ cell subpopulation.

\section{Flow cytometry analysis of additional stemness cell markers}

To investigate whether other putative stem cell markers were expressed in HNSCC cells, the mesenchymal stem cell markers, CD29 (B1-integrin), CD73 (5'-nucleotidase), CD90 (Thy-1), and CD105 (Endogin) were selected and analyzed by flow cytometry. CD29 expression showed the strongest correlation with the CD44 expression. Almost all cells (99.6\%) were CD29 and CD44 double-positive. Only $\sim 6 \%$ of the cells were CD29+ and CD24+, the same percentage found for CD24+/CD44+ cells (Figure 8A). CD73 also showed a strong correlation with CD44 expression. Approximately $92 \%$ of the cells were CD73 and CD44 double-positive, while only $\sim 6 \%$ of the cells were CD73+/CD24+, similar to CD24+/CD44+ cells (Figure 8B). 


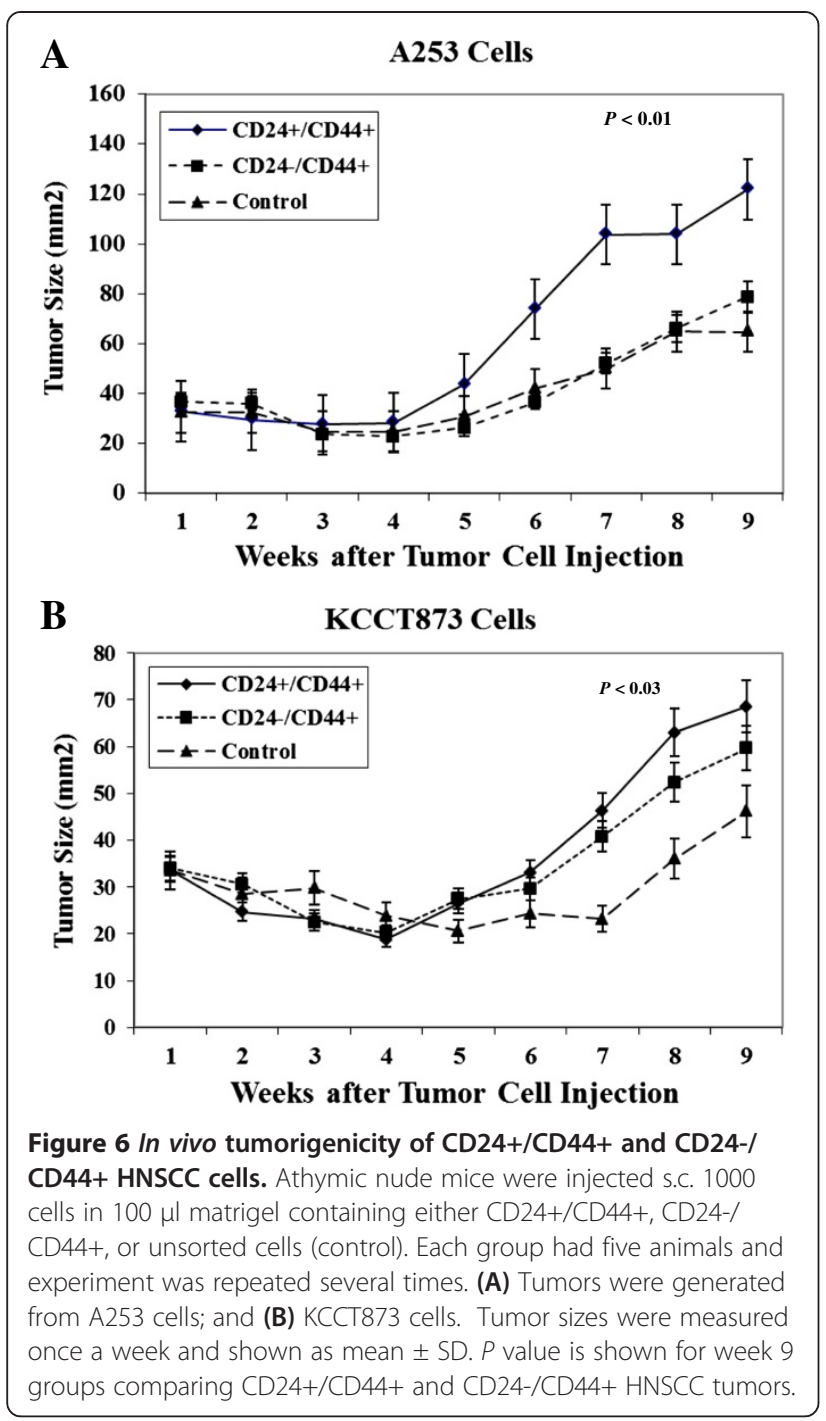

On the other hand, neither CD90 nor CD105 showed any correlation with either CD24 or CD44 expression (data not shown).

\section{Discussion}

We have identified and characterized a distinct CD24+ subpopulation in the CD44+ population of HNSCC tumors. These CD24+/CD44+ cells derived from HNSCC cell lines displayed several features typically seen in cancer stem cells, including the ability to differentiate and self-renewal. CD24+/CD44+ cells were more proliferative and invasive in vitro and more tumorigenic in vivo forming larger tumors in immunodeficient mice compared to its counterpart CD24-/CD44+ cells. In addition, CD24+/CD44+ cells were slightly more resistant to chemotherapeutic agents compared to CD24-/CD44+ cells. These findings indicate that a distinct CD24+/CD44+ subpopulation may represent CSC or tumor initiating cells in HNSCC.

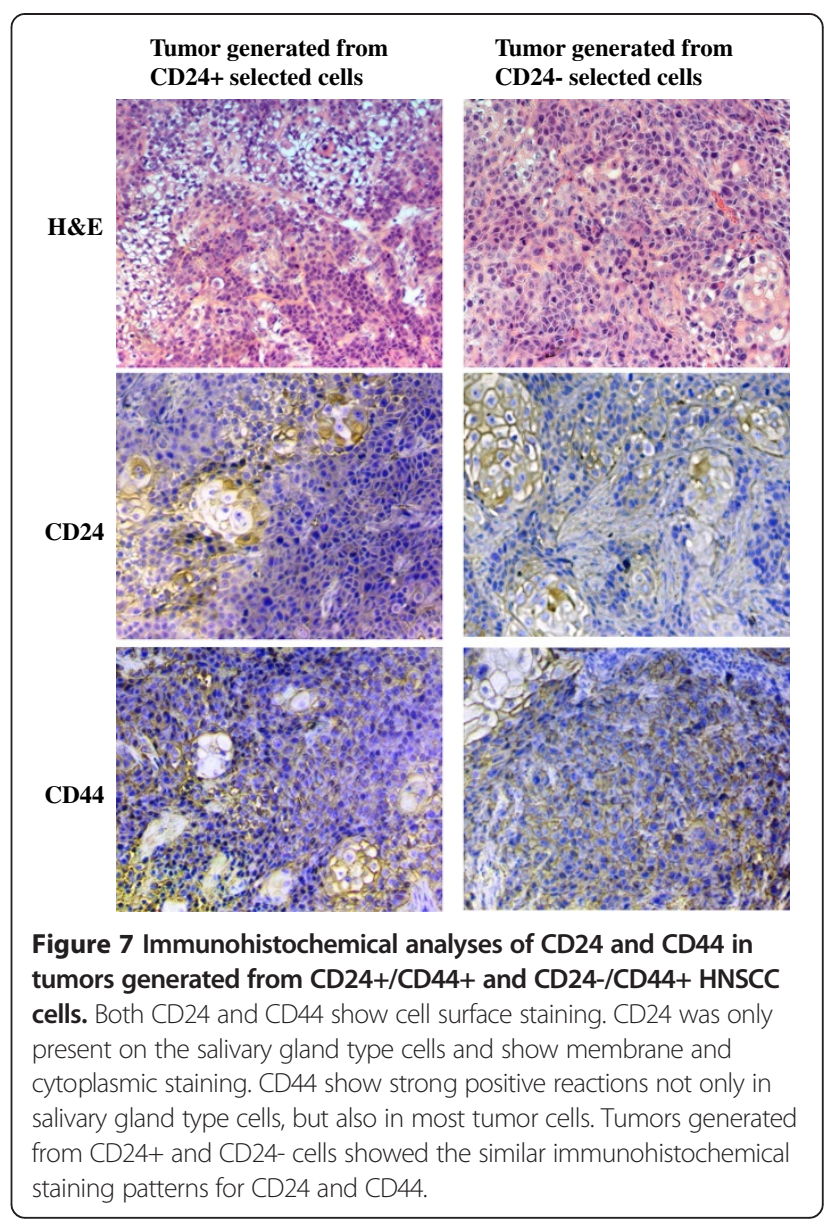

We confirmed the stemness feature of CD24+/CD44+ cells by showing that CD24+/CD44+ cells express higher levels of BMI1 and Nanog genes compared to CD24-/ CD44+ cells. BMI1 has been shown to play a role in the self-renewal of hematopoietic stem cells [14] and is considered a stem cell related gene. BMI1 has also been implicated in tumorigenesis, primarily in leukemias [13], and in several human cancers including HNSCC [12]. Similarly, Nanog gene has been shown to be associated with stemness of human embryonic stem cells [28]. These results support our finding that $\mathrm{CD} 24+/ \mathrm{CD} 44+$ cell subpopulations are indeed CSC in HNSCC. Our data also show a strong correlation between CD29 ( $\beta 1$-integrin) and CD44 expression in HNSCC. More than 99\% cells were CD29 and CD44 double-positive, indicating CD24+/ CD44+ cells were also CD29+. Recently, a subpopulation of cells ( $\left.\mathrm{Lin}^{-} / \mathrm{CD} 29+/ \mathrm{CD} 24+\right)$ isolated from mouse mammary cells was identified as mammary stem cells [29]. It is also reported that CD24 expression positively associated with salivary gland cancer stage III/IV [30]. These authors showed that double positive (CD24+/CD44+) cells may represent tumors with most aggressive behavior and worst prognosis [30]. 

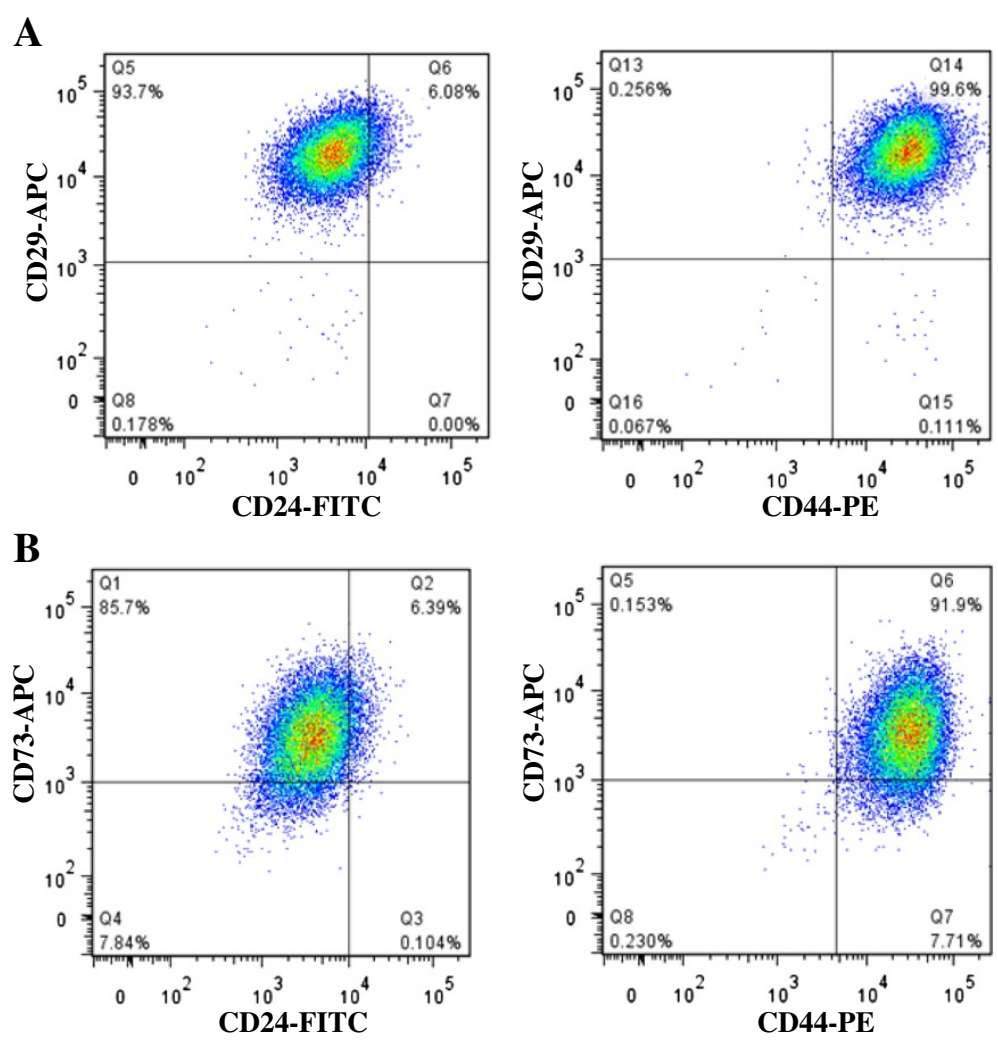

Figure 8 Representative flow cytometry plots analyzing expression of CD29, CD24, CD44, and CD73 in A253 HNSCC cells. (A) Co-expression of CD29 with CD44 and CD24. (B) Co-expression of CD73 with CD44 and CD24.

Although ALDH1, CD133, Oct3/4, and Sox2 have been identified as a putative marker for cancer stem cells in many cancers including HNSCC, we did not find a significant difference of these genes between CD24+/CD44+ and CD24-/CD44+ cell populations. In addition, Oct3/4, Sox 2 and CD133 were not consistently expressed in these cells. It is possible that different tumor cell lines, types and origin of tumors may have different phenotype of HNSCC CSCs.

Previous studies have demonstrated that CD24 is involved in cell adhesion and metastatic tumor spread $[19,31,32]$, and may be one of the cancer stem cell markers expressed in various cancer cell lines [33]. Consistent with our observations, a highly tumorigenic subpopulation of cells with CD44+/CD24+/ESA + phenotype was identified as cancer stem cells in pancreatic cancer [11]. Although this phenotype was only 0.2 to $0.8 \%$ in the whole pancreatic cancer cell population, it had a 100 -fold increased tumorigenic potential compared with other phenotypes [11]. Similarly, a CD24+/CD44+ cancer stem cell subpopulation has been identified in solid tumors and cancer cell lines in both colorectal and ovarian cancers $[8,9]$. CD24 has been shown to be related to invasiveness and differentiation of colorectal adenocarcinoma [34]. CD24 has also been identified as one of the cancer stem cell markers in human malignant mesothelioma cells [35]. These studies suggest that CD24 is both a marker of tumor aggressiveness and a promoter of metastatic tumor growth. Thus, targeting CD24 may offer new approach for therapy of human cancer including HNSCC.

Similar to CD24, previous studies have identified CD44, BMI1 and ALDH1 as putative markers for CSC in head and neck squamous cell carcinomas [12,16,17]. CD44 has also been identified as one of the CSC markers in various other cancer types $[8,11,12,20,33]$. CD44 was not only found to be constitutively expressed in the HNSCC cell lines, but also abundantly expressed in head and neck carcinomas [21,36,37]. HNSCC tumors can arise from many location of the upper aerodigestive tract, including the nasal cavity, sinus cavities, oral cavity, pharynx, or larynx. The various locations associated with malignant transformation implicated a wide-range of tumors representative of the anatomic locations [38]. Although multiple cell surface markers have been identified as cancer stem cell markers, it is clear that no marker can be used universally to identify cancer stem cells in HNSCC. Expression of various CSC markers shows great variations between different tumor types, even in the same tumor but different subtypes [33]. CD24+/CD44+ subpopulation identified in our study may represent a new subtype of the 
cancer stem cells in HNSCC, specifically in salivary gland malignant neoplasms.

It was noted that the tumors generated by both $\mathrm{CD} 24+/$ CD44+ and CD24-/CD44+ cells were positive for CD24+/ CD44+ in IHC studies. IHC staining of xenograft tumor tissues showed positive staining for CD24 on the salivary gland appearing structures. In addition, strong positive staining for CD44 was observed not only on the surface of salivary gland appearing structure, but also on the carcinoma cells within the tumor mass. There are two possible explanations for the presence of CD24+/CD44+ tumor cells from CD24-/CD44+ tumors. First, CD24+/CD44+ cells may have been generated during the in vivo tumor growth from CD24-/CD44+ cell population. This hypothesis is supported by recent publications that indicate that normal and neoplastic nonstem cells can spontaneously convert to a stem-like state. Chaffer et al., showed that $\mathrm{CD} 44^{\text {hi }}$ cells can differentiate into $\mathrm{CD} 44^{\mathrm{lo}} / \mathrm{CD} 24+/ \mathrm{ESA}^{-}$ and $\mathrm{CD} 44^{\mathrm{lo}} / \mathrm{CD} 24+/ \mathrm{ESA}+$ progeny, and $\mathrm{CD} 44^{\mathrm{lo}}$ cells can spontaneously convert to CD44 ${ }^{\text {hi }}$ cells [39]. Second, since CD24+/CD44+ and CD24-/CD44+ HNSCC cells were sorted by FACS technology, we cannot rule out the possibility of undetectable residual CD24+/CD44+ cells contaminating the CD24-/CD44+ cell population, which resulted in CD24+/CD44+ cells within the xenograft tumors, although this was considered a remote possibility.

\section{Conclusion}

We have demonstrated that HNSCC contain a distinct CD24+/CD44+ cell subpopulation that possesses cancer stem cell-like properties. CD24+/CD44+ cells are able to self-renew, differentiate into different phenotypes, initiate and develop tumors in athymic nude mice faster. Identification of cancer stem cells may provide novel insights into the development of new therapeutic approaches for HNSCC.

\section{Additional file}

Additional file 1: Table S1. Selected gene primers for $q R T-P C R$.

\section{Abbreviations}

HNSCC: Head and neck squamous cell carcinoma; CSC: Cancer stem cell; ALDH: Aldehyde dehydrogenase; BMI1: BMI1 polycomb ring finger oncogene; ESA: Epithelial-specific antigen.

\section{Competing interests}

All authors declare that they have no competing interest.

\section{Authors' contributions}

$\mathrm{JH}$ designed the study, carried out the experimental work, performed data analysis and interpreted results, and drafted the manuscript. TF and SRH carried out some experimental work, collected and analyzed data, interpreted results, and edited manuscript. RKP conceived and designed the study, supervised data analysis, interpreted results, edited and revised the manuscript, and negotiated for its publication. All authors approved the submission of this version of manuscript, and assert that the document represents valid work. All contributing authors have no disclosures to make.

\section{Acknowledgements}

We thank Howard Mostowski in the Flow Cytometry Core facility of Center for Biologics Evaluation and Research, FDA for performing fluorescent-activated cell sorting, and Drs. Steven Bauer, Robert Aksamit, and Mohammad Heidaran for reading and critiquing this manuscript.

\section{Author details}

${ }^{1}$ Tumor Vaccines and Biotechnology Branch, Division of Cellular and Gene Therapies, Center for Biologics Evaluation and Research, Food and Drug Administration, NIH Bldg 29B, Rm 2NN20, 29 Lincoln Dr., Bethesda, MD 20892, USA. ${ }^{2}$ Current address: Department of Gastroenterology, NTT Medical Center Tokyo, Tokyo, Japan.

Received: 14 July 2013 Accepted: 26 February 2014

Published: 11 March 2014

\section{References}

1. Mannelli G, Gallo O: Cancer stem cells hypothesis and stem cells ion head and neck cancers. Cancer Treat Rev 2012, 38:515-539.

2. Goon PKC, Stanley MA, Ebmayer J, Steinstrsässer L, Upile T, Jerjes W, Bernall-Sprekelsen M, Görner M, Sudhoff HH: HPV \& head and neck cancer: a descriptive update. Head Neck Oncol 2009, 1:36.

3. Marur S, Forastiere AA: Head and neck cancer: changing epidemiology, diagnosis, and treatment. Mayo Clin Proc 2008, 83:489-501.

4. Nguyen LV, Vanner R, Dirks $P$, Eaves CJ: Cancer stem cells: an evolving concept. Nat Rev Cancer 2012, 12:133-143.

5. Reya T, Morrison SJ, Clarke MF, Weissman IL: Stem cells, cancer, and cancer stem cells. Nature 2011, 414:105-111.

6. Huntly BJP, Gilliland DG: Leukaemia stem cells and the evolution of cancer-stem-cell research. Nat Rev 2005, 5:311-321.

7. Singh S, Hawkins C, Clarke ID, Squire JA, Bayanl J, Hide T, Henkelman RM Cusimano MD, Dirks PB: Identification of human brain tumor initiating cells. Nature 2004, 432:396-401.

8. Yeung TM, Gradhi SC, Wilding JL, Muschel R, Bodmer WF: Cancer stem cells from colorectal cancer-derived cell lines. PNAS 2009, 107:3722-3727.

9. Gao MQ, Choi YP, Kang S, Houn JH, Cho NH: CD24+ cells from hierarchically organized ovarian cancer are enriched in cancer stem cells. Oncogene 2010, 29:2697-80.

10. Overdevest JB, Thomas S, Kristiansen G, Hansel DE, Smith SC, Theodorescu D: CD24 offers a therapeutic target for control of bladder cancer metastasis based on a requirement for lung colonization. Cancer Res 2011, 71:3802-3811.

11. Li C, Heidt DG, Dalerba P, Burant CF, Zhang L, Adsay V, Wicha M, Clarke MF, Simeone DM: Identification of pancreatic cancer stem cells. Cancer Res 2007, 67:1030-1037.

12. Prince ME, Sivanandan R, Kaczorowski A, Wolf GT, Kaplan MJ, Dalerba P, Weissman IL, Clarke MF, Ailles LE: Identification of a subpopulation of cells with cancer stem cell properties in head and neck squamous cell carcinoma. PNAS 2007, 104:973-978.

13. Lessard J, Sauvageaun G: Bmi-1 determines the proliferative capacity of normal and leukaemic stem cells. Nature 2003, 423:255-260.

14. Park IK, Morrison SJ, Clarken MF: Bmi1, stem cells, and senescence regulation. J Clin Invest 2004, 113:175-179.

15. Harper LJ, Piper K, Common J, Fortune F, Mackenzie IC: Stem cell patterns in cell lines derived from head and neck squamous cell carcinoma. J Oral Pathol Med 2007, 36:594-603.

16. Chen YC, Chen YW, Hsu HS, Tseng LM, Huang PI, Lu KH, Chen DT, Tai LK, Yung MC, Chang SC, Ku HH, Chiou SH, Lo WL: Aldehyde dehydrogenase 1 is a putative marker for cancer stem cells in head and neck squamous cancer. Biochem Biophy Res Commun 2009, 385:307-313.

17. Clay MR, Tabor M, Owen JH, Carey TE, Bradford CR, Wolf GT, Wicha MS, Prince ME: Single-marker identification of head and neck squamous cell carcinoma cancer stem cells with aldehyde dehydrogenase. Head Neck 2010, 32:1195-1201.

18. Alberts AE, Chen C, Koberle B, Qian X, Klussmann JP, Wollenberg B, Kaufmann AM: Stem cells in squamous head and neck cancer. Crit Rev Oncol/Hemoatol 2012, 81:224-240.

19. Baumann $P$, Cremers $N$, Kroese F, Orend $G$, Chiquet-Ehrismann R, Uede $T$, Yagita H, Sleeman JP: CD24 expression causes the acquisition of multiple cellular properties associated with tumor growth and metastasis. Cancer Res 2005, 65:10783-10793. 
20. Oliveira LR, Oliveira-Costa JP, Araujo IM, Soave DF, Zanetti JS, Soares FA, Zucoloto S, Ribeiro-Silva A: Cancer stem cell immunophentypes in oral squamous cell carcinoma. J Oral Path Med 2011, 40:135-142.

21. Han J, Kioi M, Chu WS, Kasperbauer JL, Strome SE, Puri RK: Identification of potential therapeutic targets in human head \& neck squamous cell carcinoma. Head Neck Oncol 2009, 1:27.

22. Kawakami K, Leland P, Puri RK: Structure, function, and targeting of interleukin 4 receptors on human head and neck cancer cells. Cancer Res 2000, 60:2981-2987.

23. Han J, Daniel JC: Biosynthesis of type VI collagen by glioblastoma cells and possible function in cell invasion of three-dimension matrices. Connect Tissue Res 1995, 31:161-170.

24. Galluzzi L, Senovilla L, Vitale I, Michels J, Martins I, Kepp O, Castedo M, Kroemer G: Molecular mechanisms of cisplatin resistance. Oncogen 2012, 31:1869-1883.

25. Mini E, Nobili S, Caciagli B, Landini I, Mazzei T: Cellular pharmacology of gemcitabine. Ann Oncol 2006, 17:v7-v12.

26. Dean M, Fojo T, Bates S: Tumor stem cells and drug resistance. Nat Rev 2005, 5:275-284.

27. Hu G, Li F, Ouyang K, Xie F, Tang X, Wang K, Han S, Jiang Z, Zhu M, Wen D, Qin X, Zhang L: Intrinsic gemcitabine resistance in a novel pancreatic cancer cell line is associated with cancer stem cell-like phenotype. Int J Oncol 2012, 40:798-806.

28. Bhattacharya B, Milura T, Brandenberger R, Mejido J, Luo Y, Yang AX, Joshi BH, Ginis I, Thies RS, Amit M, Lyons I, Condie BG, Itskovitz-Eldor J, Rao MS, Puri RK: Gene expressions in human embryonic stem cell lines: unique molecular signature. Blood 2004, 103:2956-2964.

29. Shackleton M, Vaillsny F, Simpson KJ, Stingl J, Smyth GK, Asselin-Labbat ML, Wu L, Lindeman GJ, Visvader JE: Generation of a functional mammary gland from a single stem cell. Nature 2006, 439:84-88.

30. Soave DF, da Costa JPO, de Silveira GG, lanez RC, de Oliveira LR, Lourenco SV, Ribeiro-Silva A: CD44/CD24 immunophenotypes on clinicopathologic features of salivary glands malignant neoplasms. Diagn Patho/ 2013, 8:29.

31. Kristiansen G, Schluns K, Yongwei Y, Denkert C, Dietel M, Peterson I: CD24 expression is a new prognostic marker in breast cancer. Clin Cancer Res 2003, 9:4906-4913

32. Kristiansen G, Pilarsky C, Pervan J, Stürzebecher B, Stephan C, Jung K Loening S, Rosenthal A, Dietel M: CD24 expression is a significant predictor of PSA relapse and poor prognosis in low grade or organ confined prostate cancer. Prostate 2004, 58:183-192.

33. Stuelten CH, Mertins S, Busch II, Gowens M, Scudiero DA, Burkett MW, Hite KM, Alley M, Hollingshead M, Shoemaker RH, Niederhuber JE: Complex display of putative tumor stem cell markers in the $\mathrm{NCl} 60$ tumor cell line panel. Stem Cell 2010, 28:649-660.

34. Choi D, Lee HW, Hur KY, Kim JJ, Park GS, Jang SH, Song YS, Jang KS, Paik SS: Cancer stem cell markers CD133 and CD24 correlated with invasiveness and differentiation in colorectal adenocarcinoma. World J Gastroenterol 2009, 15:2258-2264.

35. Ghani Fl, Yamazaki H, Iwata S, Okamoto T, Aoe K, Okabe K, Mimura Y, Fujimoto N, Kishimoto T, Yamada T, Xu CW, Morimoto C: Identification of cancer stem cell markers in human malignant mesothelioma cells. Biochem Biophy res Comm 2011, 404:735-742.

36. Pries R, Wittkope $N$, Trenkle $T$, Nitsch SM, Wollenberg B: Potential stem cell marker CD44 is constitutively expressed in permanent cell lines of head and neck cancer. In vivo 2008, 22:89-92.

37. Mack B, Gires O: CD44s and CD44v6 expression in head and neck epithelia. PlosOne 2008, 3:10

38. Liebertz DJ, Lechner MG, Masood R, Sinha UK, Han J, Puri RK, Correa AJ, Epstein AL: Establishment and characterization of a novel head and neck squamous cell carcinoma cell line USC-HN1. Head Neck Oncol 2010, 2:5.

39. Chaffer CL, Bruechmann I, Scheel C, Kaestli AJ, Wiggins PA, Rodrigues LO, Brooks M, Reinhardt F, Su Y, Polyak K, Arendt LM, Kuperwasser C, Bierie B, Weinberg RA: Normal and neoplastic nonstem cells can spontaneously convert to a stem-like state. PNAS 2011, 108:7950-7955.

doi:10.1186/1471-2407-14-173

Cite this article as: Han et al:: Identification and characterization of cancer stem cells in human head and neck squamous cell carcinoma. BMC Cancer 2014 14:173.

\section{Submit your next manuscript to BioMed Central and take full advantage of:}

- Convenient online submission

- Thorough peer review

- No space constraints or color figure charges

- Immediate publication on acceptance

- Inclusion in PubMed, CAS, Scopus and Google Scholar

- Research which is freely available for redistribution 